\title{
Changing Ploidy as a Strategy: The Irish Potato Famine Pathogen Shifts Ploidy in Relation to Its Sexuality
}

\author{
Ying Li, ${ }^{1}$ He Shen, ${ }^{1}$ Qian Zhou, ${ }^{1,2}$ Kun Qian, ${ }^{1}$ Theo van der Lee, ${ }^{3}$ and Sanwen Huang ${ }^{1,2}$ \\ ${ }^{1}$ Institute of Vegetables and Flowers, Chinese Academy of Agricultural Sciences, Beijing 100081, China; ${ }^{2}$ Agricultural Genome \\ Institute at Shenzhen, Chinese Academy of Agricultural Sciences, Shenzhen 518124, China; and ${ }^{3}$ Biointeractions \& Plant \\ Health, Wageningen University \& Research Center, Plant Research International, Wageningen 6700AA, The Netherlands
}

Accepted 8 December 2016.

\begin{abstract}
The oomycete Phytophthora infestans was the causal agent of the Irish Great Famine and is a recurring threat to global food security. The pathogen can reproduce both sexually and asexually, with high potential to adapt to various environments and great risk to break disease resistance genes in potato. As are other oomycetes, $P$. infestans is regarded to be diploid during the vegetative phase of its life cycle, although some studies reported trisomy and polyploidy. Using microsatellite fingerprinting, genome-wide assessment of single nucleotide polymorphisms, nuclear DNA quantification, and microscopic counting of chromosome numbers, we assessed the ploidy level of a comprehensive selection of isolates. All progenies from sexual populations of $P$. infestans in nature were found to be diploid, in contrast nearly all dominant asexual lineages, including the most important pandemic clonal lineages US-1 and 13_A2 were triploid. Such triploids possess significantly more allelic variation than diploids. We observed that triploid genotype can change to a diploid genome constitution when exposed to artificial stress conditions. This study reveals that fluctuations in the ploidy level may be a key factor in the adaptation process of this notorious plant destroyer and imposes an extra challenge to control this disease.
\end{abstract}

The causal agent of the Irish famine in the 1840s, Phytophthora infestans continues to be a threat to potato and tomato. Even today, late blight caused by $P$. infestans remains the most destructive disease on potato, the world's third largest food crop. Understanding the success of this pathogen and its mechanisms of rapid adaption is of critical importance for disease management. $P$. infestans is a classic example of a pathogen that combines the advantages of a large genotype diversity generated in sexual crosses with a large population expansion via rapid asexual cycles of successful genotypes (McDonald and Linde 2002).

Although sexual offspring were found in the field in many regions of the world ( $\mathrm{Li}$ et al. 2012; Sjöholm et al. 2013), studies indicate that the majority of late blight infections are caused by just a few clonal lineages that spread over large geographic areas, sometimes extending over multiple continents

Y. Li and H. Shen contributed equally to this work.

Corresponding authors: Y. Li; E-mail: liying05@caas.cn; and S. Huang; E-mail: huangsanwen@caas.cn

*The $\boldsymbol{e}$-Xtra logo stands for "electronic extra" and indicates that three supplementary figures, five supplementary tables, and three supplementary data files are published online.

(c) 2017 The American Phytopathological Society
(Li et al. 2012). Examples are the oldest recorded clonal lineage HERB-1, which was dominant in both Europe and North America between 1845 and 1896 and triggered the Irish famine (Yoshida et al. 2013); subsequently, the US-1 clonal lineage of the A1 mating type first emerged in North America in the 20th century and dominated the globe (Martin et al. 2013; Yoshida et al. 2013). Recently (in the 1990s), the dominance of the US-1 clonal lineage collapsed and appears to be replaced by more aggressive lineages, such as 13_A2 in Europe and Asia (Cooke et al. 2012; Li et al. 2013b) as well as US-22 (A2) in North America (Danies et al. 2014; Hu et al. 2012). Evolutionary theory predicts that, due to the lack of recombination, accumulation of deleterious mutations would eventually lead to extinction of asexual lineages (Muller 1964). However, for P. infestans, we observed that such successful clonal lineages disperse over a wide geographical area for decades, challenging Muller's law.

A possible solution is the occurrence of polyploidization. In several studies trisomy, aneuploidy, and polyploidy in $P$. infestans were observed (Carter et al. 1999; Hamed and Gisi 2013; Yoshida et al. 2013), although P. infestans generally is regarded as autodiploid (Grünwald and Flier 2005). In plants, polyploidy can enhance vigor, because of multiallelic interactions, and a buffer for the mutational load in asexual reproduction by masking deleterious alleles, as reported in fungi, plants, and animals (Simon et al. 2003; Kearney. 2005; Li et al. 2012). Could polyploidy be an adaptive strategy for $P$. infestans? Population surveys of $P$. infestans, performed in many countries using a highly efficient and standardized multilocus marker set, resulted in a comprehensive global view on the $P$. infestans population structure (Delgado et al. 2013; Li et al. 2012, 2013b). We re-analyzed the data from these previous surveys, adopting allele dosage strategies to determine to what extent polyploidy occurs in $P$. infestans. Besides this multilocus genotyping, we performed resequencing of selected isolates, flow cytometry (FLC), and microscopic observations to determine the polyploidy level of isolates. To further demonstrate the role of polyploidy in adaption ability, isolates with different ploidy levels were cultured under stress conditions and the fate of ploidy level was determined. Our results provide more insights into the genomic consequences and adaptive advantages of ploidy transitions for $P$. infestans.

\section{RESULTS}

Microsatellite analysis suggests ploidy variation relative to reproduction ways.

In previous studies, we used multiplex microsatellites (12-plex simple sequence repeats [SSRs]) to fingerprint $520 P$. infestans isolates and defined the population structure, including 397 
isolates from nine major clonal lineages on four continents and 123 isolates from three sexual populations from The Netherlands, Mexico, and Tunisia (Delgado et al. 2013; Li et al. 2012, 2013b). These previous analyses were based on the allele size, although clear differences of allele dosage were noted. To survey the occurrence and frequency of polyploidy in the nine major clonal lineages, we analyzed the fingerprint dataset of individual isolates by scoring the allele dosage, as shown by the peak height at each SSR locus (Table 1; Supplementary Fig. S1; Supplementary Table S1; Supplementary Data S1). For eight of nine asexual lineages, we observed a high ratio of triallelic loci $(0.73$ to 0.99$)$, whereas the value ( 0.14 to 0.35$)$ in three sexual populations is significantly lower $(P=0.008)$. Nearly all dominant clonal lineages were found to be triploid, while all isolates derived from sexual populations were found to be diploid.

\section{Resequencing, FLC, and microscope observation confirm the ploidy variation in $P$. infestans.}

To further inspect the genome-wide ploidy level, we selected 12 representative isolates from a meta-population in The Netherlands that included three asexual lineages (NL 13_A2, NL 06_A1, and NL 08_A1) and two sexual populations (NL pop2 and NL pop3) (Table 2). Resequencing of representative isolates generated a total of 147 billion base pairs of sequence, with a median depth of $50 \times$ and coverage of $76 \%$ of the assembled T30-4 genome. Sequences were aligned against the T30-4 reference genome to identify single nucleotide polymorphisms (SNPs). Relative read depth at each heterozygous SNP was used to determine the ploidy level of the isolates. For a diploid genome, the mean of read counts at heterozygous positions should have a single mode at 0.5 , for which the allele ratio is $1: 1$. For a triploid genome, two modes, 0.33 and 0.67 (1:2 and 2:1) are expected (Yoshida et al. 2013, Supplementary Table S2). Consistent with the SSR analyses, all nine isolates from the sexual populations displayed the diploid mode, whereas the three isolates from the asexual lineages (NL07041, NL08080, and NL08452) showed the triploid mode (Table 2; Fig. 1; Supplementary Fig. S2). We also re-analyzed genome sequences of seven isolates from a previous report (Yoshida et al. 2013) (Table 2; Fig. 1), which confirmed the triploid nature of all four isolates from asexual lineages US-1 and EC-1. No tetraploid isolates, giving rise to allelic ratios of $1: 3$ or $3: 1$, were identified. This confirmed that dominant clonal lineages are triploid while isolates derived from sexual populations are diploid.

Table 1. The allele dosage of microsatellite loci in asexual and sexual populations

\begin{tabular}{|c|c|c|c|c|c|c|c|c|c|}
\hline Line & Area & $\mathbf{M T}^{\mathbf{a}}$ & mtDNA $^{b}$ & No. isolate & No. locus & 3 -allele loci ${ }^{c}$ & 2-allele locic & 1-allele loci ${ }^{c}$ & 3-allele ratio ${ }^{d}$ \\
\hline \multicolumn{10}{|l|}{ Asexual lineages } \\
\hline CN Northern & Northern China & A1 & IIa & 67 & 10 & 106 & 213 & 346 & 0.33 \\
\hline CN 13_A2 & Southwest China & A2 & Ia & 64 & 10 & 375 & 25 & 240 & 0.94 \\
\hline CN Fujian & Eastern China & A1 & $\mathrm{IIb}$ & 36 & 10 & 153 & 17 & 190 & 0.90 \\
\hline NL 13_A2 & The Netherlands & A2 & Ia & 73 & 12 & 591 & 5 & 280 & 0.99 \\
\hline NL 06_A1 & The Netherlands & A1 & $\mathrm{Ib}$ & 7 & 12 & 52 & 5 & 17 & 0.91 \\
\hline NL 08_A1 & The Netherlands & $\mathrm{A} 1$ & Ia & 16 & 12 & 91 & 15 & 47 & 0.86 \\
\hline EC-1 & Ecuador & A1 & IIa & 73 & 12 & 422 & 124 & 330 & 0.77 \\
\hline TU-1 & Tunisia & A1 & Ia & 57 & 12 & 312 & 114 & 251 & 0.73 \\
\hline US-1 & United States & A1 & $\mathrm{Ib}$ & 4 & 12 & 25 & 9 & 14 & 0.74 \\
\hline \multicolumn{10}{|l|}{ Sexual population } \\
\hline NL pop2 & The Netherlands & $\mathrm{A} 1, \mathrm{~A} 2$ & Ia, IIa & 37 & 10 & 45 & 83 & 241 & 0.35 \\
\hline Mex & Mexico & $\mathrm{A} 1, \mathrm{~A} 2$ & & 44 & 10 & 25 & 154 & 253 & 0.14 \\
\hline TU-2 & Tunisia & $\mathrm{A} 1, \mathrm{~A} 2$ & Ia & 42 & 10 & 56 & 105 & 238 & 0.35 \\
\hline
\end{tabular}

\footnotetext{
a Mating type.

${ }^{\mathrm{b}}$ Mitochondria haplotype.

c 3-allele, 2-allele, and 1-allele means tri-, bi-, and mono-allelic, respectively.

d 3-allele loci/(3-allele loci +2 -allele loci).
}

Table 2. Ploidy analysis of isolates by genome resequencing, flow cytometry, and microscope observation

\begin{tabular}{|c|c|c|c|c|c|c|c|}
\hline Isolate & $\mathbf{M T}^{\mathbf{a}}$ & Line & Seq. depth & DNA content $(p g)^{b}$ & No. of chromosomes & Ploidy mode & Reference \\
\hline NL00150 & A1 & NL pop3 & 53 & $\mathrm{Nd}$ & $13.5 \pm 1.0$ & Diploid & This study \\
\hline NL04092 & A1 & NL pop3 & 54 & $\mathrm{Nd}$ & $\mathrm{Nd}$ & Diploid & This study \\
\hline NL04106 & A2 & NL pop3 & 39 & $\mathrm{Nd}$ & $\mathrm{Nd}$ & Diploid & This study \\
\hline NL05159 & $\mathrm{A} 1$ & NL pop3 & 51 & $\mathrm{Nd}$ & $14.2 \pm 0.8$ & Diploid & This study \\
\hline NL05385 & A1 & NL pop3 & 55 & $\mathrm{Nd}$ & $\mathrm{Nd}$ & Diploid & This study \\
\hline NL05387 & A1 & NL pop3 & 51 & $\mathrm{Nd}$ & $13.2 \pm 0.9$ & Diploid & This study \\
\hline NL05890 & A2 & NL pop3 & 40 & $\mathrm{Nd}$ & $12.8 \pm 0.8$ & Diploid & This study \\
\hline NL07434 & A2 & NL pop2 & 54 & $0.56 \pm 0.02$ & $14.2 \pm 0.7$ & Diploid & Yoshida et al. 2013 \\
\hline NL08797 & A2 & NL pop2 & 23 & $0.56 \pm 0.01$ & $13.8 \pm 0.4$ & Diploid & This study \\
\hline P17777 & A1 & $\mathrm{Nd}$ & 48 & $\mathrm{Nd}$ & $\mathrm{Nd}$ & Diploid & Yoshida et al. 2013 \\
\hline $06 \_3928 A^{c}$ & $\mathrm{~A} 2$ & NL 13_A2 & 50 & $\mathrm{Nd}$ & $\mathrm{Nd}$ & Triploid $^{\mathrm{c}}$ & Yoshida et al. 2013 \\
\hline DDR7602 & A1 & US-1 & 13 & $\mathrm{Nd}$ & $\mathrm{Nd}$ & Triploid & Yoshida et al. 2013 \\
\hline LBUS5 & A1 & US-1 & 9 & $\mathrm{Nd}$ & $\mathrm{Nd}$ & Triploid & Yoshida et al. 2013 \\
\hline NL07041 & A2 & NL 13_A2 & 106 & $0.81 \pm 0.03$ & $20.7 \pm 0.4$ & Triploid & This study \\
\hline NL08080 & A1 & NL 08_A1 & 43 & $0.82 \pm 0.03$ & $20.3 \pm 0.9$ & Triploid & This study \\
\hline NL08452 & A1 & NL 06_A1 & 41 & $0.83 \pm 0.02$ & $20.8 \pm 0.4$ & Triploid & This study \\
\hline P13527 & A1 & $\mathrm{EC}-1$ & 20 & $\mathrm{Nd}$ & $\mathrm{Nd}$ & Triploid & Yoshida et al. 2013 \\
\hline P13626 & A1 & EC-1 & 34 & $\mathrm{Nd}$ & $\mathrm{Nd}$ & Triploid & Yoshida et al. 2013 \\
\hline
\end{tabular}

\footnotetext{
a Mating type.

${ }^{\mathrm{b}} \mathrm{Nd}=$ Not determined.

c 06_3928A appeared to be tetraploid in previous research (Yoshida et al. 2013), but our analysis suggests that it is triploid.
} 
We also measured the DNA content using FLC (Supplementary Fig. S3). Isolate NL07434 (mating type A2), a representative from a sexual mating population, was selected as a diploid reference genome, as it had been investigated previously (Yoshida et al. 2013) as well as in this study. The DNA content of another isolate, NL08797 (A2), derived from the sexual mating population, was highly similar compared with the diploid reference, indicating that this isolate is also diploid. In contrast, the DNA
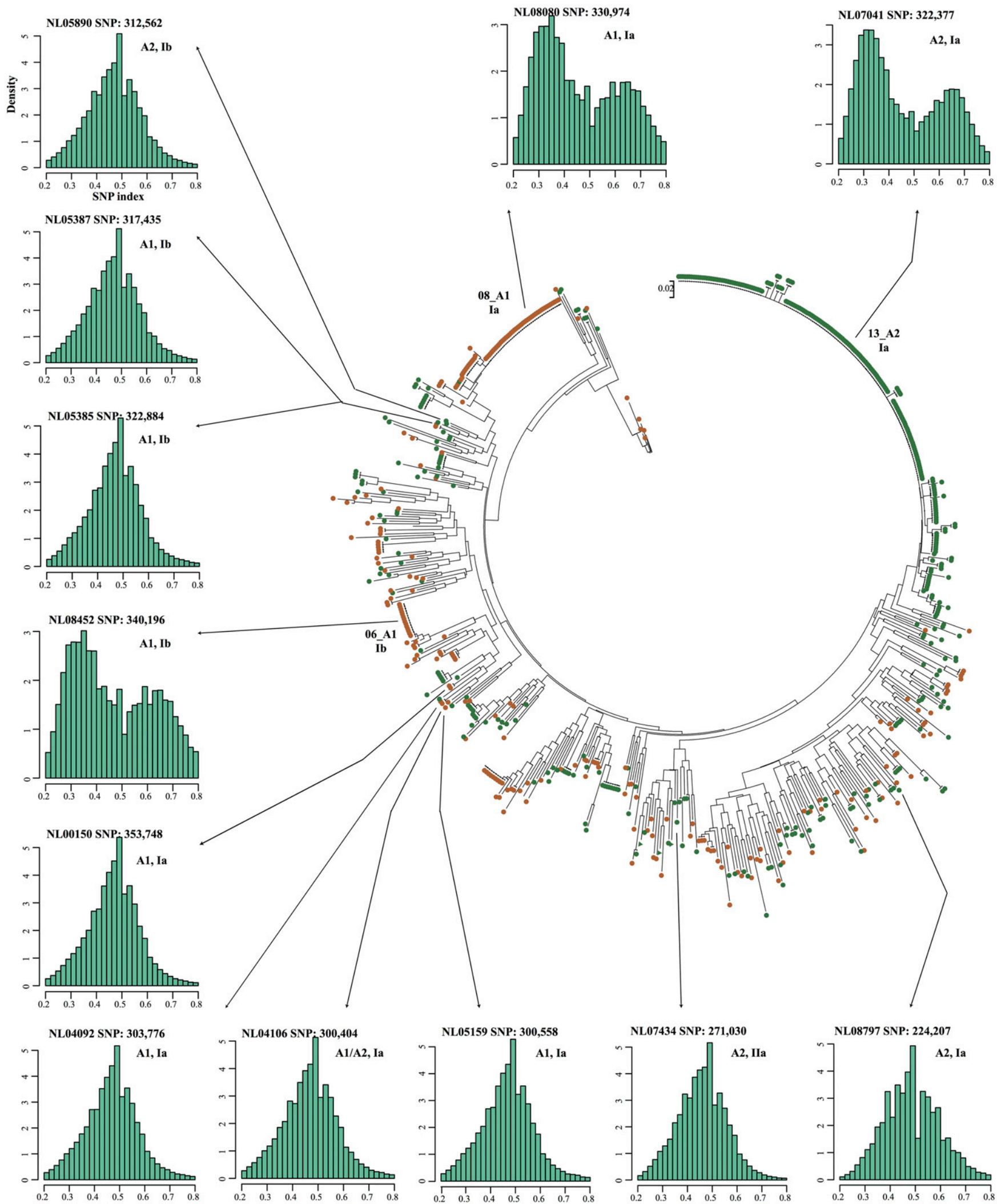

Fig. 1. Sexuality and ploidy. The nuclear phylogeny generated in a previous study (Li et al. 2012) was used for sampling strategy. Three representative isolates (NL07041, NL08080, and NL08452) were selected from three major clonal lineages in The Netherlands and nine sexual isolates were from genetic neighbor clades. The ploidy mode was determined with the mean frequency of read counts at heterozygous positions, a single and diploid mode at 0.5 , while two modes, 0.33 and 0.67 , were for triploid genomes. 
content of the asexual isolates NL08452 (A1) and NL07041 (A2), representing the asexually propagating populations, was about 1.5 times that of NL07434 and NL08797, consistent with their triploid genome. No heterokaryons were found in this study.

Finally, we counted chromosome numbers under the microscope; the average chromosome number for isolates representing the sexually propagating populations was 13.6 , as opposed to an average of 20.6 chromosomes per nucleus for isolates representing the asexual clonal lineages (Table 2, Supplementary Table S3).

\section{Triploids preserve more variation than diploids.}

As nearly all successful clonal lineages in P. infestans appear to be triploid, we studied the genetic variation between diploid and triploid genotypes to understand if triploid genotypes could harbor more genetic variation that could be beneficial in adaptation. The total number of SNPs did not show a significant difference between diploids and triploids (Table 3; Supplementary Table S4). We explored heterozygous SNPs in the complete nuclear genome, which showed that triploid genotypes contained $13 \%$ more heterozygous SNPs than diploid genotypes. A highly significant difference $(P<0.0001, t$ test $)$ was also observed when the same analysis was performed in coding regions. A further extension involving the same analysis on the large repertoire of putative effector proteins, such as RXLR and CRN genes, showed average frequencies of heterozygous SNPs are 0.74 and 0.77 , respectively, in triploid genotypes and 0.62 and 0.69 for diploid genotypes. This difference is significant $(P<0.002$ and $P<0.002$, $t$ test, respectively) (Table 3 ). These data demonstrate that triploids do possess a higher level of functional variation and do maintain more mutations in genomic, genic, and candidate effector genes.

\section{Triploids show genome plasticity under stressful conditions.}

We hypothesized that adverse conditions and environmental stresses may result in genome instability of triploids. To test this hypothesis, a series of stress induction experiments on two diploid (NL07434 and NL08797) and four triploid isolates

Table 3. Frequency of heterozygous single nucleotide polymorphisms ${ }^{\mathrm{a}}$

\begin{tabular}{lccccc}
\hline & & & \multicolumn{3}{c}{ Regions } \\
\cline { 4 - 6 } Isolate & Ploidy & Whole genome & Coding & RxLR & CRN \\
\hline NL00150 & 2 & 0.69 & 0.7 & 0.74 & 0.72 \\
NL04092 & 2 & 0.6 & 0.6 & 0.59 & 0.68 \\
NL04106 & 2 & 0.6 & 0.6 & 0.59 & 0.67 \\
NL05159 & 2 & 0.61 & 0.63 & 0.56 & 0.67 \\
NL05385 & 2 & 0.62 & 0.62 & 0.57 & 0.66 \\
NL05387 & 2 & 0.62 & 0.62 & 0.57 & 0.65 \\
NL05890 & 2 & 0.62 & 0.61 & 0.61 & 0.67 \\
NL07434 & 2 & 0.67 & 0.64 & 0.69 & 0.79 \\
NL08797 & 2 & 0.61 & 0.62 & 0.57 & 0.67 \\
JW11-8 & 2 & 0.67 & 0.67 & 0.69 & 0.73 \\
CN-SC30 & 3 & 0.73 & 0.76 & 0.7 & 0.7 \\
CN-SC39 & 3 & 0.74 & 0.76 & 0.71 & 0.73 \\
NL02123 & 3 & 0.79 & 0.81 & 0.78 & 0.77 \\
NL08080 & 3 & 0.8 & 0.82 & 0.78 & 0.8 \\
06_3928A & 3 & 0.75 & 0.77 & 0.73 & 0.79 \\
CN152 & 3 & 0.75 & 0.77 & 0.72 & 0.75 \\
P13527 & 3 & 0.75 & 0.75 & 0.75 & 0.82 \\
P13626 & 3 & 0.79 & 0.82 & 0.77 & 0.81 \\
NL08452 & 3 & 0.78 & 0.79 & 0.8 & 0.79 \\
NL07041 & 3 & 0.73 & 0.75 & 0.69 & 0.75 \\
NL07434-M & 2 & 0.66 & 0.63 & 0.65 & 0.77 \\
NL07434-S & 2 & 0.68 & 0.64 & 0.67 & 0.8 \\
NL08452-M & 2 & 0.67 & 0.66 & 0.63 & 0.75 \\
NL08452-S & 3 & 0.81 & 0.8 & 0.83 & 0.81 \\
\hline a Genome sizes: whole genome & $228,543,505$ bp, coding region & $26,332,808$ \\
bp, RxLR region & 315,029 bp, and CRN region & $=693,952$ bp. & \\
& & & & &
\end{tabular}

(CN39, CN152, NL08080, and NL08452) were performed with eight passages and 10 days per passage. After 80 days of continuous exposure to environmental stress, the genotypic variation and the ploidy level of the asexual single-spore progeny was determined by multiplex 12 SSRs (Supplementary Data S2). Among these treatments, the most genotypic variation was identified on two specific stress conditions, a sublethal dose of metalaxyl $(20 \mu \mathrm{g} / \mathrm{ml}$ in rye medium) and a low carbon medium (rye medium without additional sugar). To determine any change in ploidy, we selected one diploid (NL07434) and one triploid (NL08452) and performed whole-genome resequencing to compare the ploidy variation in the diploid and triploid ancestors with one daughter isolate. Subsequently, an induced diploid clone (NL08452-M) was identified, derived from the triploid isolate NL08452 after treatment with $20 \mu \mathrm{g} / \mathrm{ml}$ metalaxyl (Fig. 2). The frequency of heterozygous SNPs in NL08452-M decreased from $78 \%$, observed in the triploid NL08452, to $67 \%$. The frequency of heterozygous SNPs in NL08452-M is similar to diploid genotypes found in the field (Fig. 2; Table 3).

To further identify the mechanism by which the triploid isolate reduced its ploidy, we compared all identified SNPs before and after treatments and counted the number of SNPs lost and the appearance of novel SNPs (Supplementary Table S5). The stressinduced diploid isolate NL08452-M lost $40 \%$ of all heterozygous SNPs of the triploid parental isolate NL08452 (Fig. 3). However, the frequency of novel SNPs in NL08452-M was around 30\%, which is similar to other investigated diploid or triploid isolates (Fig. 2), indicating the whole process of chromosome loss happened rapidly and ploidy reduction should occur just before or at the beginning of mutation accumulation.

For all heterozygous SNPs, we tried to computationally calculate the haplotypes phase, using heterozygosity events flanking each SNP. A triploid genome constitution has three haplotypes. Depending on its origin, this can be either "aa'b" or "abc". Both NL08452-M and NL08452-S (under the condition of no additive sugar in rye medium) accumulated a similar frequency of novel SNPs $(P<0.05)$ compared with NL08452, illustrating each haplotype was not evolved independently, as would be expected for an "abc" genome constitution. Inspection of SNP haplotypes across the genome identified 13,075 three-SNP phasing regions, $71 \%$ of which displayed the transition pattern of "aa'b" to "ab" (Fig. 3). To map and localize the density and distribution of haplotype changes on the genome, the 51 supercontigs longer than $1 \mathrm{Mb}$ from the T30-4 reference assembly were screened individually. In $66 \%$ of inspected supercontigs the AAB genome constitution of NL08452 had changed to AB in NL08452-M (Fig. 3), demonstrating that the triploid NL08452 lost nearly a complete chromosomal set but mostly kept the heterozygosis as natural diploids.

\section{DISCUSSION}

In genetic studies of $P$. infestans, its genome constitution should no longer be simplified as diploid. This study underlines the importance of the assessment of the ploidy level of the isolates studied. Isolates with a triploid genome constitution typify the successful global epidemic of pandemic asexual lineages. The most successful clonal lineages, such as US-1, NL 13_A2, TU-1, NL 06_A1, and NL 08_A1, are all triploid. The triploid genome constitution could result from (partly) unreduced gametes during meiosis in $2 \mathrm{n} \times 2 \mathrm{n}$ crosses giving rise to trisomic and triploid progeny, as was previously observed at low frequency in the sexual offspring of P. infestans (Carter et al. 1999; Hamed and Gisi 2013; van der Lee et al. 2004) and was also observed in plants and yeast (Ramanna and Jacobsen 2003; Mason and Pires 2015). Alternatively, triploids could be the result of polyploidization of a $2 n$ genotype ( $a b)$ to $4 n$ (aabb) 

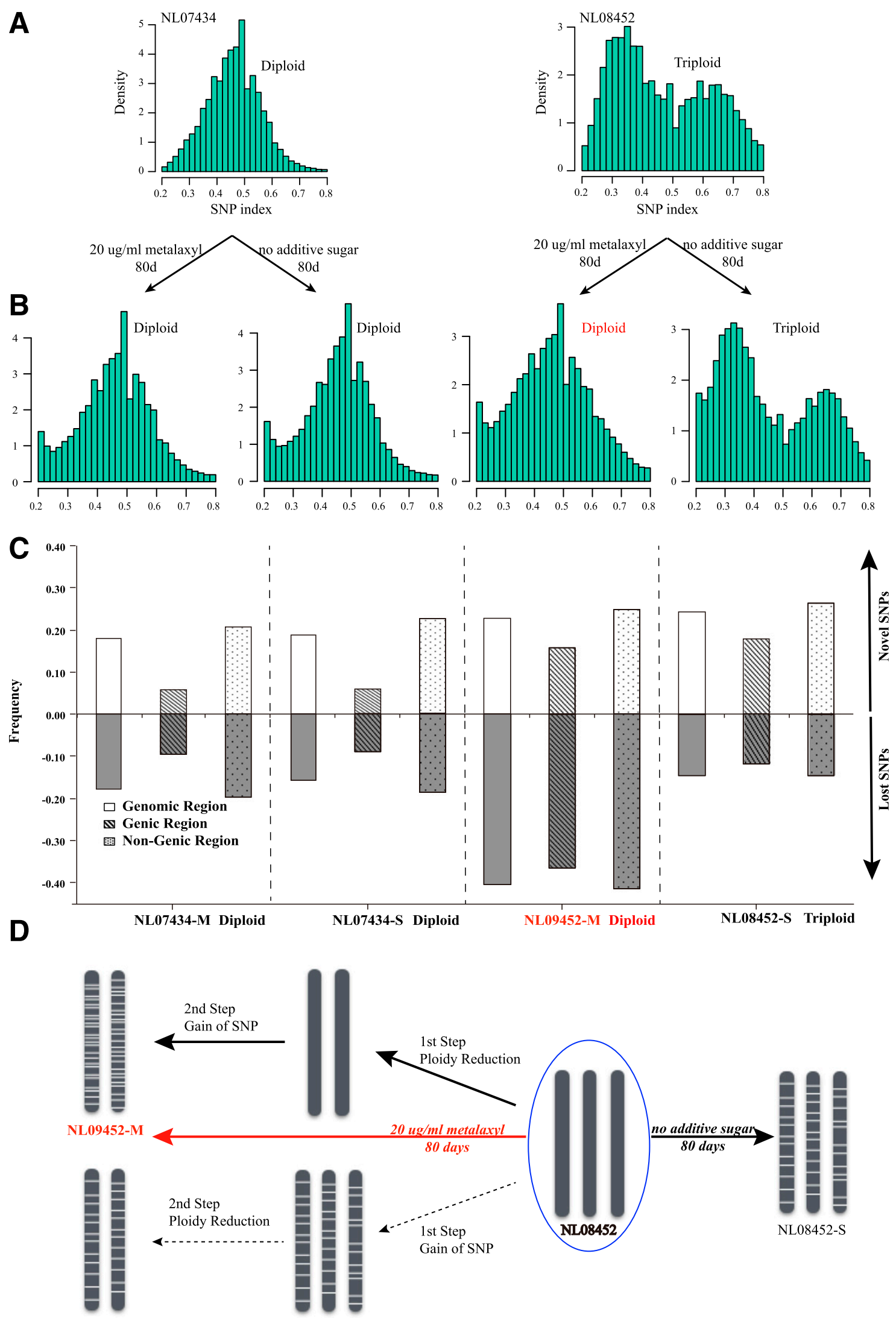

Fig. 2. Genome-wide variations after stress conditions. A, One diploid and triploid isolates were treated with $20 \mu \mathrm{g}$ of metalazyl per milliliter and no additive sugar in media for 80 days. B, Ploidy reduction in NL08452 after the treatment of metalazyl. C, The frequencies of lost single nucleotide polymorphisms (SNPs) (below 0.00) in NL08452-M are dramatically more than those in other isolates while, surprisingly, it gains no fewer novel SNPs (above 0.00) than others. D, The mechanism of ploidy reduction is proposed. When NL08452 suffered unfavorable conditions, chromosome loss happened rapidly and its ploidy was reduced just before or at the beginning of mutation accumulation. 
followed by a ploidy reduction to aab. Our results on i) ploidy reduction found in the change from $3 n$ to $2 n$, ii) the fact that we observed the aab genotype and not an abc genotype, and iii) the much higher frequency of triploids found in the successful clonal lineages may point to this alternative theory for the occurrence of triploids in nature. Triploidization in P. infestans could enhance fitness by increasing heterzygosity at an allelic level and is endowed with more adaptive plasticity than diploids, as was reported for yeast, in which tetraploids showed a higher beneficial mutation rate than haploid and diploid strains (Selmecki et al. 2015). In addition, to increase heterzygosity, triploidization may support superior fitness caused by gene dosage and multi-allelism. Asexual reproduction can allow triploid clones to rapidly disseminate over large geographic areas with little burden of the accumulation of deleterious mutations that would eventually lead to extinction of strictly asexual propagation (Muller 1964).

Nevertheless, a sexual cycle could be an advantage under specific conditions, in which case a triploid genome constitution could cause problems because of unbalanced gametes and progeny. Exposure to adverse growth conditions may promote genome instability, which may induce the return to diploid genotypes that could be more successful to generate a sexual offspring in which the genome-wide variation could be recombined and create new combinations better adapted and may survive the adverse conditions (Berman and Hadany 2012; Otto 2008). This study shows that a triploid genotype is able to rapidly return to a diploid state that may be better suited for sexual reproduction with other isolates with a 2 ngenetic constitution. Rapid chromosome loss leading to ploidy reduction has also been inferred in the yeast

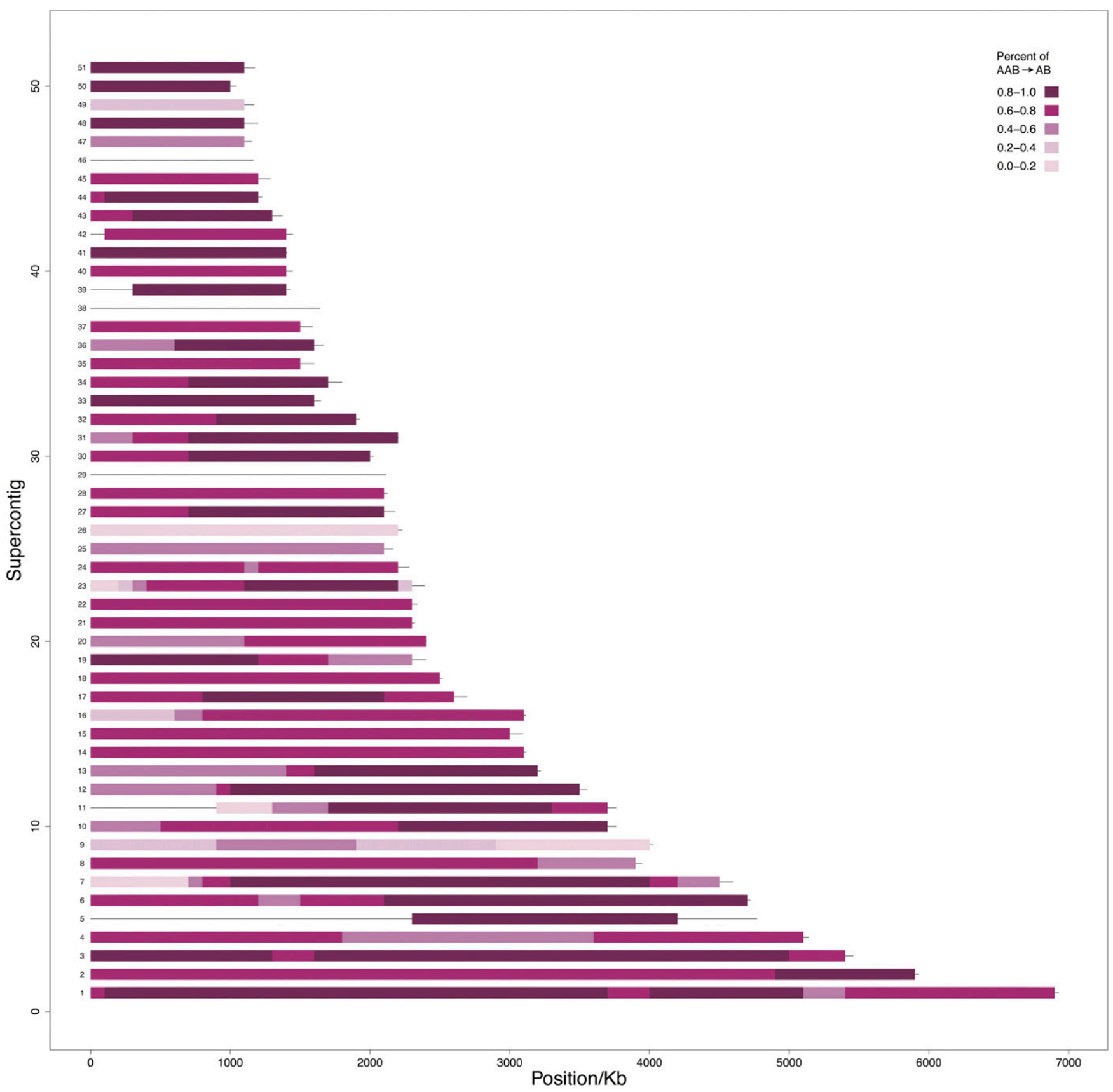

Fig. 3. The distribution of three-SNP (single nucleotide polymorphism) phasing regions in the genome of NL08452-M. Haplotype ratio of the transition pattern of "aa'b" to "ab" in a sliding window (1-Mb window at 100-Kb steps) was plotted. The graph only included supercontigs longer than $1 \mathrm{Mb}$. Full lines in the supercontigs indicate the regions without any identified three-SNP haplotypes. 
species Candida albicans (Bennett and Johnson 2003). Interestingly, $P$. infestans appears to maintain genome-wide heterozygosity after the ploidy reduction, as in most cases, a change from $\mathrm{AAB}$ to $\mathrm{AB}$ was observed. The isolate that underwent this ploidy reduction did not show obvious reduction in growth or fitness. This study provides insights into the genomic consequences of ploidy transition and adaptive advantages. We propose that, in the field, sexual diploid offspring switch to triploid during their successful vegetative lifecycles, vice versa, a triploid isolate could potentially reduce its ploidy level in order to survive under adverse conditions. The findings add a new dimension to the possibilities of survival of the pathogen during the vegetative stage at triploid level and in the sexual stage at the diploid level. Previously, genetic studies in $P$. infestans were hampered by the variable and sometimes extremely low germination rates of oospores (Grünwald and Flier 2005) in controlled crosses that impaired the establishment of sufficiently large sexual populations. The assessment of the ploidy level prior to the selection of the parental strains may increase the number of viable progeny, as crossing of isolates with a difference in ploidy levels may result in nonviable progeny. If the dominant clonal lineages are triploid and unable to generate viable progeny with diploid isolates, this may also explain why, in most regions, we either find sexual propagation or asexual (clonal) propagation. Diploid progeny derived from a cross would be good partners for sexual crosses in the next season. In contrast clonal lineages would be unsuitable as partners in sexual crosses, except when this ploidy is reduced, for instance, as a response to adverse growth conditions. This knowledge is important for future investigation of this pathogen and its practical management, highlighting the difference between sexual and asexual populations in the field and the extra risk that stress factors, including fungicide application, may have on the genome dynamics of $P$. infestans.

\section{MATERIALS AND METHODS}

\section{Definition of asexual and sexual populations and isolate collection.}

In this study, isolates from China (Li et al. 2013b), The Netherlands (Li et al. 2012), Ecuador (Delgado et al. 2013), Tunisia (Harbaoui et al. 2014), and Mexico were investigated (Table 2). The clonal lineages and sexual populations have been defined by multilocus genotype (MLG) in those previous studies. We adopted the definition of clonality widely accepted in papers dealing with the population structure of pathogens (Tibayrenc and Ayala 2012). The definitions of asexuality (clonal lineage) and sexuality (sexual population) in this study do not refer to the cytological mechanism of reproduction but, rather, to the population structure that results from an absence or restriction of genetic recombination. The asexuality obtains wherever isolates with the same mating type show MLGs that are identical or nearly identical. In contrast sexual progeny shows extensive recombination of alleles.

\section{Microsatellite analysis.}

Twelve microsatellite markers were used (Knapova and Gisi 2002; Lees et al. 2006; Li et al. 2010, 2013a). Amplification of the SSR markers was carried out as described by Li et. al (2013a). The amplicon was capillary-electrophoresed on an automated ABI 3730, according to the manufacturer's instructions. SSR allele sizing and height were performed using GeneMapper v3.7 software (Applied Biosystems).

\section{Resequencing data and SNP calling.}

The reference genome sequence of the artificial strain T30-4 and the resequencing data used in this study were published in previous studies (Cooke et al. 2012; Haas et al. 2009; Martin et al. 2013;
Yoshida et al. 2013). The method of read mapping was used as described (Yoshida et al. 2013). The resequenced isolates selected from previous studies were re-analyzed. The reference genome has 4,921 supercontigs, many of which are too small to allow for reliable genotyping. Therefore, the largest 100 supercontigs, representing $162 \mathrm{Mb}$ of genomic sequence, were analyzed.

The isolates were sequenced using the Illumina Hi-Seq 2000 sequencer. The sequencing generated, on average, 100 million 100-bp paired-end reads for each isolate. The sequencing reads of each isolate were mapped to the T30-4 reference genome using BWA (Li and Durbin. 2009) and SNP calling was conducted subsequently, using Sequence Alignment/Maptools (Li et al. 2009). Several criteria were considered in SNP filtering: i) a SNP should be biallelic between the isolate and T30-4 genomes; ii) at the SNP locus, the phred quality score of base sequencing and score of read mapping should both be higher than $30 ;$ i) each allele of a SNP should be supported by at least four reads; and iv) the nonreference allele frequency should be between 0.2 and 0.8 at a heterozygous SNP locus (Yoshida et al. 2013).

\section{FLC and microscopy observation of chromosomes.}

The nuclei were collected by simply chopping hyphae with a scalpel blade in phosphate buffered saline, as described previously (Catal et al. 2010). In addition, we used zoospores for FLC. Zoospores were induced from sporangiospores by incubation with water at $4^{\circ} \mathrm{C}$ for $2 \mathrm{~h}$. The nuclei were stained by $10 \mu \mathrm{g}$ of propidium iodide per milliliter (Sigma). The samples were delivered into a laser BD FACSCalibur Flow Cytometer for data analysis.

The young hyphae stained by $50 \mu \mathrm{g}$ of propidium iodide per milliliter (Sigma) were transferred to a microscope slide for observation. The chromosomes were drawn on the paper and were counted. The observation was performed with a Zeiss LSM 780 laser scanning confocal microscope.

\section{Stress experiment.}

The series of stress experiments included fungicide stress (metalaxyl, 5 and $20 \mu \mathrm{g} / \mathrm{ml}$ ) and growth stress (low and high $\mathrm{pH}$, no additive sugar). Experiments were carried out in three duplicates for each treatment. The isolates were cultured under 80-day continuous stress exposure and, then, 20-day regular postculture for recovery and stabilization.

To minimize unstable SNPs in determination of lost and novel SNPs, one criterion of SNP calling had been changed compared with SNP calling in resequencing data and the nonreference allele frequency was between 0.25 and 0.85 at a heterozygous SNP locus. SNPs of NL07434 and NL08452 were recalled.

\section{Local haplotype phasing.}

The haplotype phasing was performed using local SNP linkage (Yoshida et al. 2015). All heterozygous SNPs in diploid NL08452-M were chosen as seed SNP. The short DNA sequencing reads were aligned to the reference genome. Subsequently, the reads were connected in haplotypes, using heterozygous SNPs. Mate reads from $5^{\prime}$ and $3^{\prime}$ reads formed right and left regions, respectively. Flanking regions were inspected for seed SNPs. If both left and right regions had seed SNPs, a three-SNP phasing region was formed. Only three seeds were identified, the whole region covering the three seeds was recorded. At this three-SNP phasing region, two haplotypes were determined. The third haplotype was calculated from triploid NL08452 by SNP index values. Here, we mainly use the term "haplotype" to describe a three-SNP phasing region. We analyzed the haplotypic change from triploid to diploid at each three-SNP region. The occurrences of losing one copy of homozygous subgenome were counted. The ratio of this expected condition in a $1-\mathrm{Mb}$ window was calculated with each slide (100 kb) by custom python script (Supplementary Data S3). 


\section{ACKNOWLEDGMENTS}

We thank G. J. T. Kessel and G. B. M. van den Bosch (Wageningen University) for providing P. infestans isolates. We appreciate Z. Zhang, B. Xie and S. Dong for useful discussion and E. Jacobsen (Wageningen University) and T. Städler (ETH Zurich) for critical reading. This work was supported by Chinese Special Fund for Agro-scientific Research in the Public Interest (201303018), National High Technology Research and Development Program (2013AA102603), National Natural Science Foundation of China (31201257), Ministry of Finance in China (1251610601001), Science and Technology Innovation Program of Chinese Academy of Agricultural Sciences (CAAS-ASTIP-IVFCAAS).

\section{LITERATURE CITED}

Bennett, R. J., and Johnson, A. D. 2003. Completion of a parasexual cycle in Candida albicans by induced chromosome loss in tetraploid strains. EMBO J. 22:2505-2515.

Berman, J., and Hadany, L. 2012. Does stress induce (para)sex? Implications for Candida albicans evolution. Trends Genet. 28:197-203.

Carter, D. A., Buck, K. W., Archer, S. A., Van der Lee, T., Shattock, R. C. and Shaw, D. S. 1999. The detection of nonhybrid, trisomic, and triploid offspring in sexual progeny of a mating of Phytophthora infestans. Fungal Genet. Biol. 26:198-208.

Catal, M., King, L., Tumbalam, P., Wiriyajitsomboon, P., Kirk, W. W., and Adams, G. C. 2010. Heterokaryotic nuclear conditions and a heterogeneous nuclear population are observed by flow cytometry in Phytophthora infestans. Cytometry A 77A:769-775.

Cooke, D. E., Cano, L. M., Raffaele, S., Bain, R. A., Cooke, L. R., Etherington, G. J., Deahl, K. L., Farrer, R. A., Gilroy, E. M., Goss, E. M., Grünwald, N. J., Hein, I., MacLean, D., McNicol, J. W., Randall, E., Oliva, R. F., Pel, M. A., Shaw, D. S., Squires, J. N., Taylor, M. C., Vleeshouwers, V. G., Birch, P. R., Lees, A. K., and Kamoun, S. 2012. Genome analyses of an aggressive and invasive lineage of the Irish potato famine pathogen. PLoS Pathog. 8:e1002940.

Danies, G., Myers, K., Mideros, M. F., Restrepo, S., Martin, F. N., Cooke, D. E., Smart, C. D., Ristaino, J. B., Seaman, A. J., Gugino, B. K., Grünwald, N. J., and Fry, W. E. 2014. An ephemeral sexual population of Phytophthora infestans in the Northeastern United States and Canada. PLoS One 9:e116354.

Delgado, R. A., Monteros-Altamirano, A. R., Li, Y., Visser, R. G. F., van der Lee, T. A. J., and Vosman, B. 2013. Large subclonal variation in Phytophthora infestans populations associated with Ecuadorian potato landraces. Plant Pathol. 62:1081-1088.

Grünwald, N. J., and Flier, W. G. 2005. The biology of Phytophthora infestans at its center of origin. Annu. Rev. Phytopathol. 43:171-190.

Haas, B. J., Kamoun, S., Zody, M. C., Jiang, R. H., Handsaker, R. E., Cano, L. M., Grabherr, M., Kodira, C. D., Raffaele, S., Torto-Alalibo, T., Bozkurt, T. O., Ah-Fong, A. M., Alvarado, L., Anderson, V. L., Armstrong, M. R., Avrova, A., Baxter, L., Beynon, J., Boevink, P. C., Bollmann, S. R., Bos, J. I., Bulone, V., Cai, G., Cakir, C., Carrington, J. C., Chawner, M. Conti, L., Costanzo, S., Ewan, R., Fahlgren, N., Fischbach, M. A. Fugelstad, J., Gilroy, E. M., Gnerre, S., Green, P. J., Grenville-Briggs, L. J., Griffith, J., Grünwald, N. J., Horn, K., Horner, N. R., Hu, C. H., Huitema, E., Jeong, D. H., Jones, A. M., Jones, J. D., Jones, R. W., Karlsson, E. K., Kunjeti, S. G., Lamour, K., Liu, Z., Ma, L., Maclean, D., Chibucos, M. C., McDonald, H., McWalters, J., Meijer, H. J., Morgan, W., Morris, P. F., Munro, C. A., O’Neill, K., Ospina-Giraldo, M., Pinzón, A., Pritchard, L., Ramsahoye, B., Ren, Q., Restrepo, S., Roy, S., Sadanandom, A., Savidor, A., Schornack, S., Schwartz, D. C., Schumann, U. D., Schwessinger, B., Seyer, L., Sharpe, T., Silvar, C., Song, J., Studholme, D. J., Sykes, S., Thines, M., van de Vondervoort, P. J., Phuntumart, V., Wawra, S., Weide, R., Win, J., Young, C., Zhou, S., Fry, W., Meyers, B. C., van West, P., Ristaino, J., Govers, F., Birch, P. R., Whisson, S. C., Judelson, H. S., and Nusbaum, C. 2009. Genome sequence and analysis of the Irish potato famine pathogen Phytophthora infestans. Nature 461:393-398.

Hamed, B. H., and Gisi, U. 2013. Generation of pathogenic F1 progeny from crosses of Phytophthora infestans isolates differing in ploidy. Plant Pathol. 62:708-718.

Harbaoui, K., Hamada, W., Li, Y., Vleeshouwers, V. G. A. A., and van der Lee, T. 2014. Increased difficulties to control late blight in Tunisia are caused by a genetically diverse Phytophthora infestans population next to the clonal lineage NA-01. Plant Dis. 98:898-908.

Hu, C.-H., Perez, F. G., Donahoo, R., McLeod, A., Myers, K., Ivors, K., Secor, G., Roberts, P. D., Deahl, K. L., Fry, W. E., and Ristaino, J. B. 2012. Recent genotypes of Phytophthora infestans in the Eastern United
States reveal clonal populations and reappearance of mefenoxam sensitivity. Plant Dis. 96:1323-1330.

Kearney, M. 2005. Hybridization, glaciation and geographical parthenogenesis. Trends Ecol. Evol. Amst. 20:495-502.

Knapova, G., and Gisi, U. 2002. Phenotypic and genotypic structure of Phytophthora infestans populations on potato and tomato in France and Switzerland. Plant Pathol. 51:641-653.

Lees, A. K., Wattier, R., Shaw, D. S., Sullivan, L., Williams, N. A., and Cooke, D. E. L. 2006. Novel microsatellite markers for the analysis of Phytophthora infestans populations. Plant Pathol. 55:311-319.

Li, H., and Durbin, R. 2009. Fast and accurate short read alignment with Burrows-Wheeler transform. Bioinformatics 25:1754-1760.

Li, H., Handsaker, B., Wysoker, A., Fennell, T., Ruan, J., Homer, N., Marth, G., Abecasis, G., Durbin, R., and 1000 Genome Project Data Processing Subgroup. 2009. The sequence alignment/map format and SAMtools. Bioinformatics 25:2078-2079.

Li, Y., Cooke, D. E. L., Jacobsen, E., and van der Lee, T. 2013a. Efficient multiplex simple sequence repeat genotyping of the oomycete plan pathogen Phytophthora infestans. J. Microbiol. Methods 92:316-322.

Li, Y., Govers, F., Mendes, O., Testa, A., Jacobsen, E., Huang, S. W., and van der Lee, T. A. J. 2010. A new set of highly informative SSR markers for Phytophthora infestans population analysis assembled into an efficient multiplex. Mol. Ecol. Resour. 10:1098-1105.

Li, Y., van der Lee, T., Zhu, J. H., Jin, G. H., Lan, C. Z., Zhu, S. X., Zhang, R. F., Liu, B. W., Zhao, Z. J., Kessel, G., Huang, S. W., and Jacobsen, E. 2013b. Population structure of Phytophthora infestans in China-Geographic clusters and presence of the EU genotype Blue_13. Plant Pathol. 62: 932-942.

Li, Y., van der Lee, T. A., Evenhuis, A., van den Bosch, G. B., van Bekkum, P. J., Förch, M. G., van Gent-Pelzer, M. P., van Raaij, H. M., Jacobsen, E., Huang, S. W., Govers, F., Vleeshouwers, V. G., and Kessel, G. J. 2012. Population dynamics of Phytophthora infestans in The Netherlands reveals expansion and spread of dominant clonal lineages and virulence in sexual offspring. G3 (Bethesda) 2:1529-1540.

Martin, M. D., Cappellini, E., Samaniego, J. A., Zepeda, M. L., Campos, P. F., Seguin-Orlando, A., Wales, N., Orlando, L., Ho, S. Y., Dietrich, F. S., Mieczkowski, P. A., Heitman, J., Willerslev, E., Krogh, A., Ristaino, J. B., and Gilbert, M. T. 2013. Reconstructing genome evolution in historic samples of the Irish potato famine pathogen. Nat. Commun. 4:2172.

Mason, A. S., and Pires, J. C. 2015. Unreduced gametes: Meiotic mishap or evolutionary mechanism? Trends Genet. 31:5-10.

McDonald, B. A., and Linde, C. 2002. Pathogen population genetics, evolutionary potential, and durable resistance. Annu. Rev. Phytopathol. 40:349-379.

Muller, H. J. 1964. The relation of recombination to mutational advance. Mutat. Res. 1:2-9.

Otto, S. P. 2008. Sexual reproduction and the evolution of sex. Nature Education 1:182.

Ramanna, M. S., and Jacobsen, E. 2003. Relevance of sexual polyploidization for crop improvement-A review. Euphytica 133:3-8.

Selmecki, A. M., Maruvka, Y. E., Richmond, P. A., Guillet, M., Shoresh, N., Sorenson, A. L., De, S., Kishony, R., Michor, F., Dowell, R., and Pellman, D. 2015. Polyploidy can drive rapid adaptation in yeast. Nature 519:349-352

Simon, J. C., Delmotte, F., Rispe, C., and Crease, T. 2003. Phylogenetic relationships between parthenogens and their sexual relatives: The possible routes to parthenogenesis in animals. Biol. J. Linn. Soc. 79: 151-163.

Sjöholm, L., Andersson, B., Högberg, N., Widmark, A. K., and Yuen, J. 2013. Genotypic diversity and migration patterns of Phytophthora infestans in the Nordic countries. Fungal Biol. 117:722-730.

Tibayrenc, M., and Ayala, F. J. 2012. Reproductive clonality of pathogens: A perspective on pathogenic viruses, bacteria, fungi, and parasitic protozoa. Proc. Natl. Acad. Sci. U.S.A. 109:E3305-E3313.

van der Lee, T., Testa, A., Robold, A., van't Klooster, J., and Govers, F. 2004. High-density genetic linkage maps of Phytophthora infestans reveal trisomic progeny and chromosomal rearrangements. Genetics 167:1643-1661

Yoshida, K., Sasaki, E., and Kamoun, S. 2015. Computational analyses of ancient pathogen DNA from herbarium samples: Challenges and prospects. Front. Plant Sci. 6:771.

Yoshida, K., Schuenemann, V. J., Cano, L. M., Pais, M., Mishra, B., Sharma, R., Lanz, C., Martin, F. N., Kamoun, S., Krause, J., Thines, M., Weigel, D., and Burbano, H. A. 2013. The rise and fall of the Phytophthora infestans lineage that triggered the Irish potato famine. eLife 2:e0731. 